\title{
Current Scientific and Regulatory Approaches for Development of Orally Inhaled and Nasal Drug Products: Overview of the IPAC-RS/University of Florida Orlando Inhalation Conference
}

\author{
Guenther Hochhaus, ${ }^{1}$ Craig Davis-Cutting, ${ }^{2}$ Martin Oliver, ${ }^{3}$ Sau L. Lee, ${ }^{4}$ and Svetlana Lyapustina ${ }^{5,6,7}$
}

Received 29 January 2015; accepted 26 May 2015; published online 2 June 2015

\begin{abstract}
This article summarizes discussions at the March 2014 conference organized by the University of Florida (UF) and International Pharmaceutical Aerosol Consortium on Regulation and Science (IPAC-RS), entitled “Orlando Inhalation Conference: Approaches in International Regulation.” The special focus of the conference was on global scientific and regulatory issues associated with the testing and demonstration of equivalence for the registration of orally inhaled drug products (OIDPs) in the United States, Europe, Brazil, China, and India. The scope included all types of OIDPs throughout their lifecycle, e.g., innovator/brand-name products, generics, modifications due to lifecycle management, device changes, etc. Details were presented for the U.S. "weight of evidence approach" for registration of generic products (which includes demonstration of in vitro and in vivo equivalence, as well as quantitative and qualitative sameness, and device similarity). The European "stepwise" approach was elucidated, and the thinking of regulatory agencies in the major emerging markets was clarified. The conference also highlighted a number of areas that would benefit from further research and discussion, especially around patient/device interface and human factor studies, statistical methods and criteria for demonstrating equivalence, the relative roles of in vivo and in vitro tests, and appropriate designs and metrics for in vivo studies of inhaled drugs.
\end{abstract}

KEY WORDS: bioequivalence; emerging markets; generics; MDIs and DPIs; regulatory science.

\section{INTRODUCTION}

OIDPs are a staple of medical treatment for patients with respiratory conditions. Yet because most of these drugs are designed to act locally in the respiratory tract and because the dose delivered to the patient depends on the patient's inhalation technique, disease state and as well as device characteristics, the suite of tests, and requirements for demonstrating safety and effectiveness of OIDPs is still an

This article reflects the views of the authors and should not be construed to represent the positions or policies of the organizations with which the authors are affiliated.

${ }^{1}$ Department of Pharmaceutics College of Pharmacy, University of Florida, Gainesville, Florida, USA.

${ }^{2}$ Research and Development, Catalent Pharma Solutions, Morrisville, North Carolina, USA.

${ }^{3}$ Vectura, Ltd., Chippenham, Wiltshire, UK.

${ }^{4}$ Office of Pharmaceutical Science, Center for Drug Evaluation and Research, US Food and Drug Administration, Silver Spring, Maryland, USA.

${ }^{5}$ Pharmaceutical Practice Group, DBR, Washington DC, USA.

${ }^{6}$ Drinker Biddle \& Reath LLP, $1500 \mathrm{~K}$ Street NW, Washington DC, 20005-1209, USA. evolving area of regulatory science, especially for generic drug products that have to demonstrate equivalence to the innovator product. Another layer of complexity is added by the international diversity of medical and regulatory approaches towards the testing and registration of both new and generic OIDPs. The purpose of the 2014 IPAC-RS/UF conference, therefore, was to provide a public forum for discussion of the latest research and regulatory thinking regarding registration of OIDPs such as metered dose

\footnotetext{
${ }^{7}$ To whom correspondence should be addressed. (e-mail: svetlana.lyapustina@dbr.com)
}

ABBREVIATIONS: BE, bioequivalence; DPI, dry powder inhaler; EU, European Union; FDA, Food and Drug Administration (of the United States); IPAC-RS, International Pharmaceutical Aerosol Consortium on Regulation and Science; MDI, metered dose inhaler; OIDP, orally inhaled drug product; PD, pharmacodynamic; PK, pharmacokinetic; R, reference (product), e.g., innovator or original product; T, test (product), e.g., a generic or modified product; UF, University of Florida 
inhalers (MDIs) and dry powder inhalers (DPIs), including new/innovator and generic/follow-on products. The conference was characterized by its focus on regulatory approaches for obtaining market registrations and by the wide geographic scope at the center of conference discussions. Participants learned about regulatory situations in the US, European Union, Brazil, China, and India; debated designs and interpretation of the pharmacokinetic (PK) and pharmacodynamic (PD) trials of generic inhalers; discussed in vitro testing and acceptance criteria for bioequivalence (BE) demonstration; and considered human factor testing of OIDPs, which are a combination of a drug formulation and a delivery device that needs to be actuated by a patient when administering a dose.

In addition to the formal podium presentations and panel discussions, the audience actively contributed to the interactive exchange of ideas and perspectives. Overall, over 200 individuals from over 20 countries attended the conference. About $80 \%$ of all attendees were from the orally inhaled drug industry, representing 58 companies; $5 \%$ were from academia, about $7 \%$ from regulatory agencies. The rest of the attendees were consultants, press, and support staff, including several UF students with an interest in inhalation drug delivery design and testing.

A series of in-depth reports from the different sessions of the conference are presented in this theme issue of the AAPS Journal for the benefit of the wider international scientific, academic, and regulatory communities with an interest in inhalation drug products. The following review papers in this issue provide an integrated view of talks and discussions related to a given topic:

- Regulatory considerations for approval of generic inhalation drug products in the US, EU, Brazil, China, and India (by Sau Lee et al.) (1)

- Inhalation devices and patient interface: human factors (by Stefan Leiner et al.) (2)

- In vitro testing for orally inhaled products: developments in science-based regulatory approaches (by Ben Forbes et al.) (3)

- Pharmacokinetics of orally inhaled products (by Guenther Hochhaus et al.) (4)

- Pharmacodynamic studies to demonstrate bioequivalence of oral inhalation products (by Leslie Hendeles et al.) (5)

In addition, results of original research reported at the conference are presented in the following articles in this AAPS Journal issue:

- Application of the modified chi-square ratio statistic in a stepwise procedure for cascade impactor equivalence testing (by Ben Weber et al.) (6)

- Effect of device design and formulation on the in vitro comparability for multi-unit dose dry powder inhalers (by Jag Shur et al.) (7)

- In vitro, pharmacokinetic, pharmacodynamic, and safety comparisons of single and combined administration of tiotropium and salmeterol in COPD patients using different dry powder inhalers (by Stephen Horhota et al.) (8)
- Safety of $\beta 2$-agonists in asthma: linking mechanisms, meta-analyses, and regulatory practice (by Sanjeeva Dissanayake) (9).

In this overview article, we provide a succinct summary of discussions at the conference. Original presentations and program are also available on the conference's website (10).

\section{REGULATORY APPROACHES IN DIFFERENT WORLD REGIONS}

Generic medicines aim to provide more affordable, yet still safe and efficacious, high-quality alternatives to the previously approved innovator drugs. To obtain marketing authorization, the sponsor must provide to the pertinent government agency appropriate documentation about the drug's composition, manufacturing facilities, and other details helping to document and ensure the high quality of generic alternatives. Being a follow-on to a previously approved innovator drug, generics typically have an abbreviated set of data demonstrating equivalence of the generic product to a previously approved product. To that end, the generic drug sponsor must demonstrate equivalence of the proposed generic to the innovator drug through in vitro and/or in vivo studies. Both designs and results of these studies must be provided to the relevant agency for review, assessment, and approval. The types of studies, acceptance criteria, terminology (e.g., "bioequivalence" vs "therapeutic equivalence"), the overall approach, and even exact definitions of what constitutes a "generic" drug vary by the country. For example, in the U.S., the Food and Drug Administration (FDA) developed a "weight of evidence" approach, whereby equivalence in all categories of interest is required for a proposed generic drug to be approved, namely: quantitative and qualitative sameness of the formulation, similarity of the delivery device, in vitro performance and in vivo performance equivalence. By contrast, in the European Union, regulatory authorities follow a "stepwise" approach, whereby approval could be granted at the earliest step in a decision tree where equivalence is demonstrated, starting with in vitro equivalence, pharmaceutical equivalence, followed by lung deposition equivalence, systemic PK, efficacy, and, finally, safety PD equivalence. Thus, approval based on "in vitro only equivalence" is possible in the EU but not in the US.

Countries also differ in their legal systems and regulatory structure; and each country has its own system of government oversight for the registration and distribution of medical products on its territory. In the case of the European Union, in addition to each country's national governments, the Union's governing bodies provide supra-national mechanisms and requirements for OIDPs and other drugs.

The specific regulatory bodies and approval considerations in different world regions are described in detail in a separate article in this theme issue (see "Regulatory considerations for approval of generic inhalation drug products in the US, EU, Brazil, China, and India", by Sau Lee et al., AAPS Journal). A summary of specific considerations related to in vitro and in vivo tests and acceptance criteria in different region is provided in Table I. It is evident that there are some general similarities across regions but also important differences, of which product sponsors need to be well aware. 


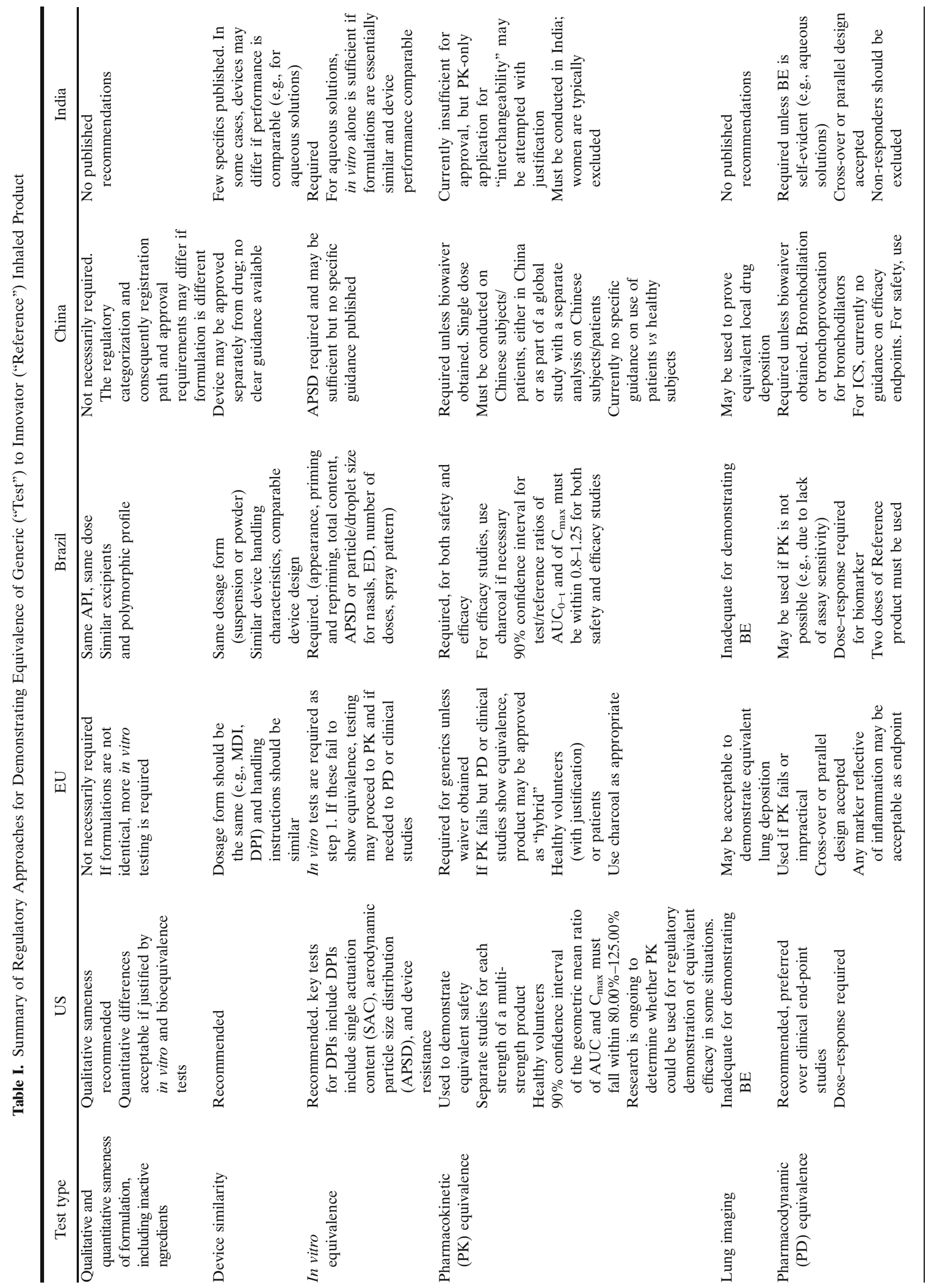




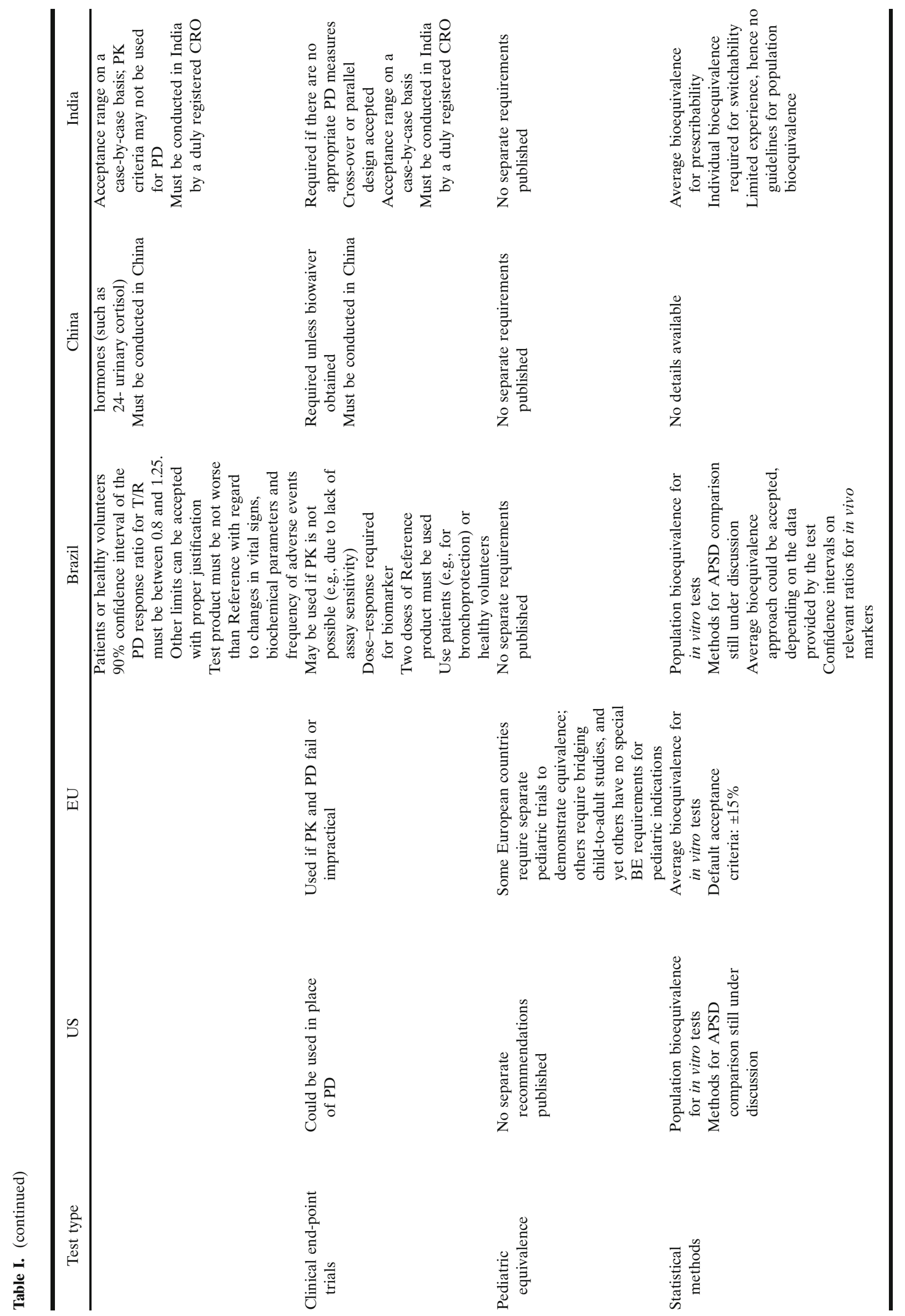


The disparities among international regulatory approaches suggest that there are ample opportunities for the regulatory and scientific communities around the world to collaborate to develop a more consistent, better aligned, science-based approach for evaluation and approval of innovator and generic OIDPs.

\section{IN VITRO AND DEVICE CONSIDERATIONS FOR DEMONSTRATING BIOEQUIVALENCE}

OIDPs present unique challenges for in vitro testing and characterization of the aerosol cloud generated and delivered by these devices. The drug dose delivered to the patient is a transient bolus of material constantly changing during the path from the inhaler to the deposition sites in the lungs. At the Orlando Inhalation Conference, the scientific and practical challenges of in vitro testing of OIDPs were considered from two perspectives: (1) the fundamental science that underpins understanding of inhaled drug delivery and the subsequent fate of drugs inhaled into the lungs, and (2) current and emerging methods for in vitro testing and their application. The conference presentations and accompanying review article (see "In vitro testing for orally inhaled products: developments in science-based regulatory approaches" by Ben Forbes et al.) discuss techniques used to assess aerosol deposition, as well as some of the factors that may impact the ability to predict the drug absorption after aerosol delivery, such as particle dissolution, permeation, particle clearance, and tissue exposure. The ability to determine an in vitro/in vivo correlation for OIDPs with various laboratory techniques remains one of the important areas for the ongoing discussion and research.

The capability to accurately measure and characterize OIDPs with in vitro test methods provide tools for establishing quality criteria for the development and regulation of both generic and innovator products. The above-mentioned article explores how companies and regulatory authorities are currently using in vitro methods to understand the formulation and device and to establish product quality. Some of the current debates about these test methods, their application, the statistics used to evaluate the data, and how all this can lead to a complex regulatory situation are also explored. It is clear from the conference that analysis and interpretation of in vitro data are increasingly important for decision-making. The article identifies a number of areas for further work to help achieve the objective of moving in vitro tests from a descriptive nature to methods that can measure product performance against objective criteria.

An additional area of complexity for OIDPs relates to the device delivering the drug to the patient. The discussion on in vitro test methods at the conference was complemented by a number of talks about challenges of device design and studying the device/patient interaction through human factors studies (2). That review article explores the importance of human factors analysis in the design process for OIDPs, the challenges which device design presents within a drug product development process, the impact of device changes during the product lifecycle from both technical and regulatory perspectives, and the difficulties of correctly using the inhalers as experienced by some patients.
Both of the referenced articles provide a practical stateof-the-art summary of the current challenges within these areas and a discussion of how techniques may be improved in the future.

\section{IN VIVO (PK, PD, AND CLINICAL ENDPOINT) CONSIDERATIONS FOR DEMONSTRATING BIOEQUIVALENCE}

Pharmacokinetic and pharmacodynamics studies are often components of OIDP bioequivalence studies to ensure equivalence in safety and efficacy between test $(\mathrm{T})$ and reference (R) inhalation products. The goal of these studies is to ensure that the pulmonary and systemic exposure (rate and extent of drug availability at the site of action and in the systemic circulation) are confirmed in an in vivo situation. Several regulatory agencies continue to put strong emphasis on such in vivo tests because in vitro/in vivo correlations (which are necessary for using in vitro assessments as substitute for in vivo studies) are not yet fully developed for OIDPs.

The PK- and PD-related presentations at the Orlando Inhalation Conference and the accompanying review articles ("PK studies to support development and approval of generic inhaled products", by Guenther Hochhaus et al., and "PD studies to demonstrate safety and efficacy of inhalation products", by Leslie Hendeles et al.) discussed a wide range of key topics, such as:

- Optimizing design of PK studies (e.g., healthy volunteers $v s$ patients; training to optimize inhalation techniques);

- Evaluating the relevance of PK and PD studies for systemic as well as pulmonary equivalence testing;

- Identifying in vitro parameters relevant for in vitro/in vivo correlations;

- Evaluating the sensitivity of PD studies for OIDPs;

- The need for harmonizing the conclusions that regulators may draw from PK studies, especially distinguishing systemic $v s$ pulmonary fate of the drug.

Most of the participants at the Orlando Inhalation Conference agreed that PK studies in healthy volunteers are the most sensitive in vivo method for assessing equivalence of systemic exposure. More importantly, the majority of participants seemed to agree that given a suitable study design (e.g., charcoal treatment for drugs that are orally available), PK results may enable comparisons of the rate and extent of respiratory drug delivery between $\mathrm{T}$ and $\mathrm{R}$ formulations.

Effects of batch-to-batch in vitro variability of $\mathrm{T}$ products on PK BE assessments was identified as an important topic for careful consideration and further research. The need for PK study designs that would allow assessment of intra-subject as well as inter-batch variability was stressed by the U.S. FDA, as this would allow incorporation of statistical methods that adjust $\mathrm{BE}$ acceptance criteria for the reference batch variability. As current in vitro studies do not seem to capture all properties affecting pulmonary fate of drug after inhalation, PK studies could be employed to obtain all relevant BE properties, such as available dose, equivalent pulmonary residence time, and central/peripheral deposition ratio. 
Presentations and discussions also addressed challenges associated with PD approaches to evaluate pulmonary and systemic BE of inhalation drugs. A particular challenge in this area is the lack of dose-response relationships for pulmonary effects of corticosteroids, including the insensitivity of biomarker studies, such as reduction of nitric oxide and airway responsiveness to adenosine for anti-inflammatory effects of corticosteroids. The OIDP experts gathered in Orlando came to the general conclusion that the currently available biomarkers for assessing pulmonary corticosteroid effects are not sensitive enough for BE studies using the dose-scaling approach. Within this context, it was also questioned what information could be drawn from parallel, single-dose clinical study designs proposed by the FDA for fluticasone propionate/salmeterol xinafoate combination formulations, which would compare potential differences on the effect-scale using doses that are close to the upper end of the $E_{\max }$ scale and are therefore always likely to pass as long as generic companies are able to afford such studies with large numbers of patients.

An 18-h area under the cortisol concentration time curve was recommended by some for determining equivalent systemic exposure of corticosteroids, while others strongly believed that PK is the most sensitive method addressing the systemic exposure of all inhaled drugs, including short- and long-acting beta- 2 adrenergic drugs. This questioned the current thinking of the European Medicines Agency, which allows approval via PD BE studies for systemic exposure by monitoring cortisol, heart rate, serum potassium, and serial electrocardiograms when $\mathrm{BE}$ is not established by PK studies.

\section{SUGGESTIONS FOR FUTURE RESEARCH AND DISCUSSIONS}

Overall, Orlando Inhalation Conference demonstrated the shared high interest in sound scientific approaches to the development and regulation of inhaled products. The presentations, panels, and audience discussions also highlighted a number of areas that merit further scientific research as well as a public clarification of the underlying principles and practical considerations. An illustrative list of such areas for further exploration is compiled below. Some of these issues may be approached differently by different countries and thus could be beneficially explored through international workshops to aid in global harmonization; while other issues should be further researched in clinics and laboratories through well-designed and subsequently published studies, in order to inform both drug development and regulatory policy.

1. What is the purpose of generic drug testing in a given world region? (And what should it be?) For example, is the goal to demonstrate identical performance or non-inferior performance of the test product compared to reference product? What weight should be given to in vitro performance $v s$ in vivo performance? Which in vitro and in vivo tests are most relevant, keeping in mind the above-mentioned overall goal of generic drug testing in a given world region?

2. Given the current diversity of legal, regulatory, cultural, and other aspects, as well as still-open scientific questions such as in vivo in vitro correlations or predictive biomarkers, to what extent could regulatory approaches across different geographic regions be harmonized, and what would be the path to harmonization?

3. Which in vitro metrics correlate to which in vivo metrics? Under what conditions does each correlation hold? How universal is each observed correlation across different product types?

4. What statistical approaches should be used to compare Aerodynamic Particle Size Distributions (APSDs) of the test and reference products, and what acceptance criteria should be applied?

5. Does testing of dissolution of inhaled drugs provide information relevant to determination of bioequivalence that cannot be obtained from testing other attributes with established methods?

6. What PK study designs could be used to compare regional lung deposition (peripheral and central) between test and reference products?

7. Drug delivery from a DPI may depend on the air flow rate generated by the patient or employed for in vitro tests. How exactly should the flow rate dependency be accounted for when comparing test and reference products?

8. How exactly should statistical approaches take into account the batch-to-batch variability of the reference product? How should such evaluations be balanced with the resources expended for testing? Should different acceptance criteria apply to drugs intended for acute use vs chronic use?

9. Does a patient's interaction with an inhaler device have a role in generic drug review, and if so, how should such patient/device interaction be tested and assessed?

10. How can both patients and health-care providers be educated on the correct inhalation technique, which is necessary for appropriate drug delivery and for minimizing variability in clinical trials? How can such an education be provided to patients outside the clinical studies and considering also that they may be using several different inhalers in parallel?

11. What $\mathrm{PD} /$ clinical study designs are most appropriate (i.e., sufficiently sensitive, discriminatory, and clinically relevant) to demonstrate bioequivalence of inhalation products? How should different patient populations and different therapeutic indications be represented in generic drug testing?

\section{CONCLUSIONS}

The 2014 UF/IPAC-RS Orlando Inhalation Conference attracted a diverse audience from around the world to discuss current approaches and challenges in developing generic OIDPs and demonstrating their equivalence in quality, safety, and efficacy to the innovator drug products. The conference highlighted general areas of alignment but also identified the need to:

- develop relevant in vitro/in vivo correlations, as current in vitro methods are not able to predict in vivo performance for OIDPs; 
- evaluate whether additional in vitro tests (e.g., dissolution tests) are necessary or relevant for the intended purpose;

- evaluate the use of PK for assessing the pulmonary fate of deposited drug (e.g., pulmonary available dose, pulmonary residence time, central to peripheral deposition) for slowly dissolving drugs;

- continue international regulatory discussions about whether PK studies are sufficient for making equivalence decisions on systemic exposure; and

- understand best ways to study human factors and device/ patient interactions, both for new and generic drugs.

\section{REFERENCES}

1. Lee SL, Saluja B, García-Arieta A, Santos GML, Li Y, Lu S, et al. Regulatory considerations for approval of generic inhalation drug products in the US, EU, Brazil, China, and India. AAPS J. 2015. doi:10.1208/s12248-015-9787-8.

2. Leiner S, Parkins D, Lastow O. Inhalation devices and patient interface: human factors. AAPS J. 2015;17(2): 457-461. doi: 10.1208/s12248-015-9717-9 http://link.springer.com/article/ 10.1208/s12248-015-9717-9. Accessed 21 Jan 2015.

3. Forbes B, Bäckman P, Christopher D, Dolovich M, Li BV, Morgan B. In vitro testing for orally inhaled products: developments in science-based regulatory approaches. AAPS J. 2015. doi: 10.1208/s12248-015-9763-3. http://www.pharmagateway.net/ ArticlePage.aspx?DOI=10.1208/s12248-015-9763-3. Accessed 19 May 2015.

4. Hochhaus G, Horhota S, Hendeles L, Suarez S, Rebello J. Pharmacokinetics of orally inhaled drug products. AAPS J. 2015. doi: 10.1208/s12248-015-9736-6. http://www.pharmagateway.net/
ArticlePage.aspx?DOI=10.1208/s12248-015-9736-6. Accessed 15 April 2015.

5. Hendeles L, Daley-Yates PT, Hermann R, Backer J, Dissanayake S, Horhota ST. Pharmacodynamic studies to demonstrate bioequivalence of oral inhalation products. AAPS J. 2015; 17 (3): 758-768. doi: 10.1208/s12248-015-9735-7. http://link.springer.com/article/ 10.1208\%2Fs12248-015-9735-7. Accessed 19 May 2015.

6. Weber B, Lee SL, Delvadia R, Lionberger R, Li BV, Tsong Y, Hochhaus G. Application of the modified chi-square ratio statistic in a stepwise procedure for cascade impactor equivalence testing. AAPS J. 2015; 17 (2): 370-379. doi: 10.1208/s12248-014-9698-0. http://www.pharmagateway.net/ ArticlePage.aspx?DOI=10.1208/s12248-014-9698-0. Accessed 15 April 2015

7. Shur J, Saluja B, Lee S, Tibbatts J, Price R. Effect of device design and formulation on the in vitro comparability for multiunit dose dry powder inhalers. AAPS J. May 2015. doi: 10.1208/ s12248-015-9775-z. http://link.springer.com/article/10.1208/ s12248-015-9775-z. Accessed 19 May 2015.

8. Horhota ST, Noord JA, Verkleij CB, Bour LJ, Sharma A, Trunk M, Cornelissen PJG. In vitro, pharmacokinetic, pharmacodynamic, and safety comparisons of single and combined administration of tiotropium and salmeterol in COPD patients using different dry powder inhalers. AAPS J. 2015. doi: 10.1208/s12248-015-9751-7. http://www.pharmagateway.net/ArticlePage.aspx?DOI=10.1208/ s12248-015-9751-7. Accessed 15 April 2015.

9. Dissanayake SB. Safety of $\beta 2$-agonists in asthma: linking mechanisms, meta-analyses and regulatory practice. AAPS J. 2015. doi: 10.1208/s12248-015-9734-8. http://www.pharmagateway.net/ ArticlePage.aspx?DOI=10.1208/s12248-015-9734-8. Accessed 15 April 2015.

10. IPAC-RS and UF. Orlando Inhalation Conference: Approaches in International Regulation. 2014. http://www.cvent.com/events/ orlando-inhalation-conference-approaches-in-international-regulation/agenda-8a749091c2c741228907ca5c70b619eb.aspx. Accessed 26 Nov 2014. 